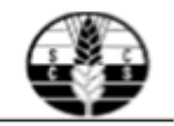

\title{
LOS SUELOS Y LA AGRICULTURA ECOLÓGICA EN LA ERA DE LA POSTVERDAD
}

Palabras-clave

Agricultura ecológica, Agricultura orgánica, Agricultura Industrial, Tecnociencia, Posverdad, Filosofía de la Ciencia, Filosofía de la tecnociencia,

\author{
Juan José lbáñez $1 \otimes$
}

\author{
$1 \mathrm{MNCN}, \mathrm{CSIC}$, Madrid \\ choloibanez \\ @hotmail.com
}

\section{RESUMEN}

Los suelos y la agricultura ecológica han sido dos temas soslayados durante décadas de la corriente principal de la literatura científica. Sin embargo, más recientemente, el reconocido fracaso de la agricultura industrial, así como la comprensión del sistema climático, han relanzado el interés por ambas disciplinas. Ya no se discute la importancia de los suelos en el funcionamiento de la biosfera, ni la necesidad de reemplazar una agricultura despilfarradora de recursos y contaminante por otra basada en genuinos principios ecológicos. Sin embargo, desde finales del siglo XX, la ciencia ha ido cambiando, tanto en sus objetivos como es su praxis, siendo paulatinamente reemplazada por lo que actualmente se denomina Tecnociencia. Esta última no persigue tanto progresar en el conocimiento del mundo, como en ser el soporte del progreso e innovación del tejido empresarial de los países, en un mundo frenéticamente competitivo a la par que insustentable. De este modo la búsqueda de la verdad ha sido reemplazada por la posverdad, en la que los científicos somos meros elementos de un sistema muy complejo, dominado por intereses económicos y geopolíticos. En este contexto, la definición de agricultura ecológica depende de los intereses de los actores implicados, relegando los aspectos técnicos a un segundo plano. En este artículo, el autor intenta discernir el futuro de una agricultura genuinamente ecológica, conforme al método científico y que necesita inexcusablemente de una gestión sostenible de los suelos, de aquellas narraciones sesgadas por intereses ajenos a la ciencia, es decir repletas de posverdades.

\section{SOILS AND ECOLOGICAL AGRICULTURE IN THE AGE OF POSTVERITY}

\section{KEYWORDS}

Organic farming Organic farming, Industrial agriculture, Technoscience, Post-truth, Philosophy of Science, Philosophy of technoscience,
SUELOS ECUATORIALES 49 (1 y 2): $96-110$

ISSN 0562-5351 e-ISSN 2665-6558

\begin{abstract}
Soils and organic farming have been two issues ignored for decades in the mainstream of scientific literature. However, more recently, the recognized failure of industrial agriculture, as well as the understanding of the climate system, have relaunched interest in both disciplines. The importance of soils in the functioning of the biosphere is no longer discussed, nor is the need to replace a wasteful agriculture of resources and pollutants with another based on genuine ecological principles. However, since the end of the twentieth century, science has been changing, both in its objectives and its praxis, being gradually replaced by what is now called Technoscience. The latter does not seek to progress so much in the knowledge of the world, as in being the support of the progress and innovation of the business fabric of the countries, in a frantically competitive and unsustainable world. In this way the search for truth has been replaced by post-truth, in which scientists are mere elements of a very complex system, dominated by economic and geopolitical interests. In this context, the definition of organic farming depends on the interests of the actors involved, relegating the technical aspects to the background. In this article, the author tries to discern the future of a genuinely organic agriculture, according to the scientific method and which inexcusably needs a sustainable management of the soils, of those narratives biased by interests outside of science, that is to say full of postverities.
\end{abstract}




\section{INTRODUCCIÓN.}

Dos avatares históricos de la carrera profesional de este autor deben terse en cuenta antes de leer el artículo. Entiendo que la mejor aportación que puedo ofrecer al lector es obligadamente heterodoxa, crítica y provocativa, más que formalmente académica, basándose en los contenidos de mi bitácora, lecturas y experiencia si bien presentaré una breve "bibliografía recomendada".

En 2005, el Subdirector General de Universidades e Investigación de la Comunidad de Madrid, tras un foro de discusión sobre el cambio climático y el protocolo de Kioto, se acercó a mí y me propuso que creara un blog en el sistema que tal CC.AA iba a inaugurar, y que a la postre recibió un importante premio Europeo. Desconocía personalmente que era esa cosa llamada blog o bitácora, por lo que desistí tantas veces como él insistió. No cejaba en su empeño, y finalmente acepté. A partir de ese instante comenzó a cambiar mi visión de la ciencia, tanto, como su valor social. Lo que realmente transformó mi perspectiva de la ciencia fue verme obligado a leer miles de noticias, relacionadas con la ciencia y la tecnología, publicadas por los más diversos actores de la sociedad. Fui comprobando que, al alejarse de los artículos y libros estrictamente científicos, y ampliar las perspectivas para introducir los factores humanos (políticos, sociales, tecnológicos, etc.), las fronteras de la indagación científica se difuminaban ante una "neblina" que "nublaba" mi entendimiento. Los investigadores somos meras marionetas en el seno de la sociedad de la posverdad y la tecnociencia, como mostraremos. Del mismo modo, durante 1987-1990, tras un accidente, los médicos entendieron que me iba a quedar irremisiblemente tullido. Recién entrado en el staff del CSIC, me vi obligado a reflexionar sobre un futuro. Casi por casualidad cayó en mis manos un libro sobre filosofía de la ciencia. Tras una placentera y estimulante lectura, seguí con la de otros muchos durante más dos años. ¿Conclusión? Los científicos no somos adiestrados en el conocimiento de los cánones que marca el método científico, sea lo que sea, por sorprendente que parezca. Mucho después impartí durante cinco años esta disciplina en un Master de la Universidad Politécnica de Madrid (UPM). Y al hacerlo pude cerciorarme que la propia ciencia estaba siendo remplazada por la tecnociencia, y la filosofía de la ciencia había dado paso a la de las tecnociencias.

\section{CIENCIA Y POSVERDAD}

Frase delo año 2016 para el Rotativo El País, conforme a Wikipedia el vocablo que da título al apartado, puede entenderse como: "Posverdad o mentira emotiva es un neologismo que describe la distorsión deliberada de una realidad, con el fin de crear y modelar la opinión pública e influir en las actitudes sociales, en la que los hechos objetivos tienen menos influencia que las apelaciones a las emociones y a las creencias personales. En cultura política, se denomina política de la posverdad (o política posfactual) a aquella en la que el debate se enmarca en apelaciones a emociones desconectándose de los detalles de la política pública y por la reiterada afirmación de puntos de discusión en los cuales las réplicas fácticas -los hechosson ignoradas. La posverdad difiere de la tradicional disputa y falsificación de la verdad, dándole una importancia "secundaria". Se resume como la idea en "el que algo aparente ser verdad es más importante que la propia verdad". Para a renglón seguido comentar que "Para algunos autores la posverdad es sencillamente mentira (falsedad) o estafa encubiertas con el término políticamente correcto de «posverdad», que ocultaría la tradicional propaganda política y el eufemismo de las relaciones públicas y la comunicación estratégica como instrumentos de 
manipulación y propaganda". La R.A.E., más brevemente define la posverdad como: "Distorsión deliberada de una realidad, que manipula creencias y emociones con el fin de influir en la opinión pública y en actitudes sociales. Los demagogos son maestros de la posverdad".

Tras cuarenta años dedicados a la indagación científica, y cinco como profesor en un máster de postgrado (UPM) en el que impartía filosofía y sociología de la ciencia, así como explicar en qué consiste el método científico he llegado a la conclusión de que efectivamente nos encontramos en la era de la postverdad. Los científicos somos un reflejo de los ciudadanos y sus valores, mientras que ambos padecemos de "malos tiempos para la lírica". Del mismo modo las autoridades de nuestras instituciones no reclaman que profundicemos en la ciencia con mayúsculas, sino que publiquemos, tanto como podamos, en revistas indexadas (a las que se "presupone" tal excelencia), pero que no es lo mismo. Y como corolario, "hoy por hoy", cualquier prolijo autor de novelas intrascendentes pero comerciales, sería

\section{CIENCIA Y TECNOCIENCIA}

Según Javier Echeverría en su "La revolución tecnocientífica": El término "tecnociencia" Io usó por primera vez Bruno Latour en 1983 para referirse a la "ciencia y tecnología". Otros muchos autores han usado ese mismo término de manera omnicomprensiva. En resumen, hoy en día, para todos, la convergencia entre ciencia y tecnología es tan profunda que todo es tecnociencia. (...). En cuanto a tradición cultural, la ciencia y la ingeniería siguen en desarrollo, aunque se ha producido una mutación, una hibridación entre ciencia y tecnología y ha surgido una nueva rama evolutiva: la tecnociencia. (...). En el caso de la tecnociencia, desde este ángulo, los objetivos de la ciencia y de la ingeniería siguen existiendo, aunque subordinados a otros, es decir, el propio conocimiento científico pasa a ser un instrumento, un medio para el logro de otros objetivos; por ejemplo, ensalzado por sus cientos de "bestsellers" ante la falta de productividad de Don Miguel de Cervantes que publicó muy pocos. ¿Y qué decir de Albert Einstein?. "Más de lo mismo". El blog o bitácora que comencé a redactar hará casi quince años, alberga ya casi 2.650 post con unas 20.000 páginas de texto alcanzando 30.000 .000 de visitas. En este medio de las redes sociales, he ido desgranando muchas ideas acerca del mundo de los suelos, pero también de las agriculturas ecológicas, conocimiento campesino, etnoagricultura y etnoedafología. Siendo cauto y calculando el número de días durante tal intervalo de tiempo, así como una lectura mínima de 30 noticias de prensa diarias, resulta que mi aparato cognitivo ha sido quebrantado por unas 142.000 , tan solo "para redactar los comentarios de mi blog". Si a ello le súmannos todo lo leído durante cuarenta años en materias relacionadas con $\mathrm{mi}$ quehacer profesional, no puedo eludir reiterar que entiendo que nos encontramos en un mundo repleto de "posverdades", así como que la ciencia ha dado paso a lo que actualmente se denomina "tecnociencia" (Echeverría, 2010).

objetivos militares, empresariales, económicos, políticos 0 sociales. La tecnociencia no la hace una sola persona, sino un equipo, una empresa, en el sentido fuerte de la palabra; se requiere una fuerte inversión para que se desarrolle la actividad tecnocientífica. Por lo tanto, la economía de la ciencia adquiere una relevancia enorme y tiene un futuro impresionante. (Uno de los rasgos distintivos de la tecnociencia es que llega la inversión privada a la investigación científica, algo sin precedentes en la historia de la ciencia.) (....) pero esta idea de que las empresas tecnocientíficas coticen en la bolsa y que llegue la inversión a la bolsa, es algo que no sucedía. (....) Este cambio rompe con la cuestión de que el científico o el ingeniero tiene que vivir del padre Estado, ahora se asume que tienen que ser extremadamente competitivos en el mercado, o sea, convertirse 
en empresa y ser capaces de atraer inversión, capital. Claro que quien invierte pide rentabilidad 0 dividendos 0 , por lo menos, capitalización en la bolsa, lo que se traduce en que la búsqueda de conocimiento y el desarrollo tecnológico están subordinados a la capitalización en bolsa 0 al aumento de los beneficios, al crecimiento del volumen de la empresa, en fin, a los objetivos típicos de la racionalidad empresarial. (....) (Echeverría, 2003).

Se trata pues de una transmutación radical por no hablar de cambio de paradigma, en el sentido kuhniano del vocablo. Si el objetivo de la ciencia consistía en hacer retroceder el muro de nuestra ignorancia acerca de la realidad del mundo, en el ámbito de la tecnociencia el conocimiento es un mero instrumento con vistas a conseguir otros objetivos menos nobles. Si la ciencia trabajaba a "hombros de gigantes", en la tecnociencia los investigadores tan solo somos simples peones en un engranaje colosal destinados al progreso tal como es entendido desde las perspectivas políticas, económicas y empresariales (Latour, 2001; Echeverría, 2007; Arana, 2009). El propósito de la ciencia consistía en ampliar nuestra visión del mundo, el de las empresas estriba en comercializar y vender (ver también el capítulo de Wikipedia).

\section{CIENCIA, TECNOCIENCIA Y AGRICULTURA ECOLÓGICA}

Desde otra perspectiva, en contra de los dictados del método científico y de la filosofía de la ciencia, una de las definiciones más frecuentes de su sociología nos informa de que "la ciencia es el acuerdo alcanzado entre científicos de prestigio". Mutatis mutandis, cuando decaen los valores y la ética del establishment, corre lo mismo con sus indagaciones. ¿Entonces?: ¿Qué es la agricultura ecológica? Pues ya pueden barruntar sus "respuestas". Empero la ciencia con mayúsculas debe ser universal. No existe una definición aceptada del concepto de agricultura ecológica, como tampoco de lo que es un suelo, por asombroso que parezca (Ibáñez et al., 2005).

Definiré pues "ad hoc" la agricultura ecológica como aquella que se sustenta exclusivamente en principios ecológicos en búsqueda de la productividad, pero también de la calidad ambiental y la salud pública. ¿Y el suelo?; ¿qué es un suelo? Podemos defender que el resultado de la alteración biogeoquímica de los materiales de la superficie terrestre, cuyas propiedades, muy concretas permiten el desarrollo de los ecosistemas terrestres tal como los conocemos hoy. Tal aparentemente inocua definición, por ejemplo, expulsa al limbo de los justos, el cultivo sobre sustratos y/o bajo plásticos, entre otros muchos sistemas agrarios cuasi-artificiales. De este modo, puede constatarse que muchos productos que se comercializan bajo la etiqueta de agricultura orgánica o ecológica no lo son, por muchos certificados que los avalen.

El comercio de productos agrícolas ecológicos se ha disparado vertiginosamente en el mundo durante las últimas décadas debido al reconocimiento de que la agricultura industrial, ha degradado y contaminado seriamente suelos, aguas y la calidad de los alimentos. La producción ecológica es ya una industria floreciente y muy rentable. Según el The World of Organic Agriculture reproducido en la siguiente página Web: "en 2016 la superficie agrícola dedicada a producción ecológica fue de 57,8 millones de hectáreas, con la participación de 2,7 millones de productores (...) en el año (...) 1999, solo se dedicaban (...) 11 millones de hectáreas y el sector tenía poco más de 200.000 productores. En cifras económicas, (....) los productos ecológicos generaron en 2016 un comercio por valor de (...) 89.700 millones de dólares (...), frente a los 17.900 millones de dólares contabilizados en el sector en el año 2000" (....) La zona geográfica con más superficie agrícola con producción ecológica reconocida es Oceanía, 
con 27,3 millones hectáreas, seguida de Europa con 13,5 millones de hectáreas, América Latina (7,1 millones de hectáreas), Asia (casi 4,9 millones de hectáreas), América del Norte (3,1 millones de hectáreas) y África (1,8 millones de hectáreas). Resulta palmario que en Latinoamérica se puede explorar y explotar esta alternativa, tanto para la alimentación de su población, como con vistas a estimular el crecimiento económico de los países, vía exportaciones. Parte de esta producción ecológica es llevada a cabo por cooperativas de productores, muchas de los cuales llegan a acuerdos con cooperativa de consumidores concienciados con su salud y la del medio ambiente. La frase "uno es lo que come" se ha popularizado entre los ciudadanos gracias al esfuerzo de las autoridades sanitarias. Por ejemplo, en Colombia, La federación Nacional de
Cafeteros (FNC) tuvo la gran idea de promocionar sus productos bajo "Juan Valdez", y tal experiencia puede ser de gran ayuda a la hora de iniciar nuevas aventuras.

Sin embargo, las grandes multinacionales se encuentran también medrando en este terreno y lo seguirán haciendo, ante la anarquía en lo concerniente a que es genuinamente ecológico y lo que no. Y como corolario, todo lo relacionado con la agricultura ecológica ya se encuentra tapizada de las posverdades y fraudes reconocidos, típicos de la tecnociencia, como nos informa, por ejemplo, el título de la siguiente nota española de prensa: "Ofensiva contra el fraude de los falsos productos bio". La única garantía de ingerir alimentos saludables estriba en las alianzas entre las asociaciones de productores y consumidores.

\section{SOSTENIBILIDAD Y TEORÍAS ECONÓMICAS: ECONOMÍA NEOCLÁSICA, BIOECONOMÍA, ECONOMÍA AMBIENTAL Y ECONOMÍA DE LOS RECURSOS NATURALES}

Cualquier debate sobre las ventajas de la agricultura ecológica respecto a las industriales, debe basarse en una serie de herramientas modelo-teóricas, previas y contrastadas. Se necesita también y ante todo partir de unos conceptos claros y precisos. Lamentablemente, la desafortunada costumbre de generar conjeturas, que raramente hipótesis, y casi ninguna teoría ampliamente corroborada, abunda de nuevo en la ceremonia de la confusión. Por ejemplo, no es lo mismo analizar la viabilidad e interés (pros y contras) de esta práctica de manejo agrario basada en principios ecológicos tomando los siguientes marcos de referencia: (i) economía neoclásica, (ii) bioeconomía, (iii) economía ambiental, (iv) economía de los recursos naturales, (iv) economía circular, etc. Del mismo modo, incluso en el seno de las teorías económicas puras, cabe diferenciar entre el pensamiento Keynesiano y el enfoque monetarista ultra-neoliberal de Milton Friedman, que ha dado lugar a la dictadura financiera que actualmente padecemos. Resulta sorprendente que Friedman recibiera el Premio Nobel por cuanto al aplicar sus recetas en Chile, Argentina, Reino Unido, etc. siempre terminaron fracasando estrepitosamente.

Actualmente, incluso la mayor parte de las instituciones internacionales consideran que el modelo económico actual resulta ser insustentable. En consecuencia, huelga hablar de desarrollo sostenible, en el seno de un sistema macroeconómico que no lo es, y más aún cuando soslaya el respeto por el ambiente y los recursos naturales. Por lo tanto, en pura lógica, este autor debería finalizar aquí el contenido del artículo. El sistema financiero actual ha mostrado ser frágil, "volátil" e impredecible, es decir de naturaleza no lineal. Los intereses cambian con demasiada rapidez como para poder adaptar numerosos aspectos de nuestras estructuras e intereses sociales a los azares económicos. Por lo tanto, apelar a vocablos como "desarrollo sostenible" en el seno ideológico de una economía basada en los postulados defendidos por los seguidores de "Milton Friedman" (denominados Chicago 
Boys) no es coherente desde ningún punto de vista. Urge pues cambiar el modelo macroeconómico vigente por otro que sea amigable con el medio ambiente, garantice la soberanía alimentaria y el bienestar de los habitantes del planeta, a la par que respete y garantice la salud de la biosfera y todos sus servicios. Sin embargo, nos topamos con otro nudo gordiano del que nadie desea hablar. Nos referimos concretamente a la demografía.

Conforme a las últimas predicciones de la ONU. Actualmente el Planeta alberga aproximadamente a 7.576.951.385 millones de personas, aumentará en 1.000.000.000 adicionales hacia 2030 , estimándose que en 2050 alcanzará la cota de 9.700 millones y finalmente de 11.200 millones a finales de siglo, en 2100. Todos reconocemos que las predicciones no son realidades, empero hacia allí nos dirigimos a no ser que una gran catástrofe natural, mega-congragación bélica 0 pandemia, acabe con buena parte de la población. Del mismo modo, según el modelo socioeconómico que impere en el futuro, las posibilidades de adaptarse "sosteniblemente" a tal número de habitantes, cambiarían. Por mucho que algunos expertos afirmen lo contrario, y en función de la rapidez de los cambios que experimenta la sociedad en todas sus facetas, se me antoja absurdo especular sobre qué tipo de "crecimiento sostenible" puede ser posible en un mundo de recursos finitos. En ausencia de cataclismos, todos tendremos que afrontar esta cruda realidad. ¡Somos demasiados! No obstante, seguimos hacia adelante, sin pensar en las consecuencias que se avecinan, teniendo en cuenta ya, entre otros factores, el grave envejecimiento de la población. Como ecólogo de formación, no puedo vislumbrar un Planeta en estado sostenible con la pirámide demográfica invertida. ¿Quién y cómo tomaría las medidas oportunas con vistas a paliar el problema? Eso sí, los fanáticos que consideran que la $\mathrm{I}+\mathrm{D}+\mathrm{i}$ resolverá nuestras tribulaciones, nos hablan ahora de paisajes de ciencia ficción a los que denominan "transhumanismo" y "post-humanismo". El lector debería inspeccionar en Internet estas descabelladas ideas, para reflexionar a renglón seguido sobre el tipo de inmortalidad que nos venden.

Y mientras tanto, soslayando la cantidad, cabría hablar del reparto espacial de los recursos humanos. Por un lado, en los países del estado de bienestar, el envejecimiento de la población ya es un serio problema, mientras que en los pobres, las criaturas abundan, mayoritariamente en condiciones infrahumanas. En sectores clave de la economía actual, los ricos disponen de la tecnología y los desheredados de las materias primas necesarias. No se trata ya de escenarios del futuro sino de síntomas alarmantemente serios. Pongamos tan solo un par de ejemplos, En Abril de 2018152 millones de criaturas sufren vejaciones como el 'trabajo infantil', reclutamiento de niños soldados, explotación sexual, ablación, matrimonios forzosos, y en general pobreza y desnutrición.

\section{DEVORANDO LOS RECURSOS EDÁFICOS Y DESIGUALDADES SOCIALES A NIVEL REGIONAL}

Del mismo modo, en los países de la opulencia, consumimos sus suelos para nuestra alimentación, bajo el aséptico vocablo de "acaparamiento de tierras". En este último ítem, los Estados desarrollados necesitan de tal abastecimiento edáfico con vistas a satisfacer sus propias necesidades $y$ crecimiento económico. Y así el "Institute for 
Advanced Sustainability Studies (IASS)" acuñó en 2006 el término de "virtual lands" ("tierras virtuales", aunque más recientemente se habla también de "la huella del suelo") al objeto de cuantificar la cantidad de recursos edáficos productivos que demanda cada país (compra 0 arrendamiento a muy largo plazo) de otros para autoabastecerse (Lugschitz et al. 2011; Würtenberger et al, 2006).

En un reciente informe de la UE, se nos informa de que (i) Europa es el continente más dependiente de la tierra (suelo) importada; (ii) Casi el $60 \%$ de la tierra (suelo) utilizada para satisfacer la demanda europea de productos agrícolas y forestales proviene de fuera del continente; (iii) Seis de los diez principales importadores de tierras son europeos: Alemania, Reino Unido, Italia, Francia, Ios Países Bajos y España; (iv) Alemania y el Reino Unido importan cada uno casi 80 millones de hectáreas al año (vi) El consumo promedio de tierra de la UE es de 1.3 hectáreas per cápita, mientras que países como China e India menor de 0.4. No debe extrañar que diversos expertos clamen que se trata de una flagrante violación de la
Declaración Universal de Derechos Humanos de las Naciones Unidas. Y el proceso continúa imparable. Parte de tal hurto global puede deberse al exceso de población en muchos países respecto a sus respectivas capacidades de carga (posibilidades), pero no resulta baladí la mala gestión de los suelos, entre las que destaca el sellado de los más fértiles del continente europeo.

Pues bien, ante tal enmarañada red de conceptos, hipótesis, datos, hechos y opiniones, típicas de la tecnociencia, la única aproximación económica que deberíamos entender no es otra que la economía ecológica, dadas las variadas pero convergentes definiciones que he detectado en la literatura. Conceptos como el de tierras virtuales o la huella del suelo, obviamente, también son muy útiles. Sin embargo, tal como se entienden actualmente, otros términos del tipo sostenibilidad, economía neoclásica, bioeconomía, economía ambiental, y más aún el manoseado "desarrollo sostenible" no dejan de ser más que ineludiblemente posverdades.

\section{AGRICULTURA ORGÁNICA, DE CONSERVACIÓN Y ECOLÓGICA.}

En las últimas décadas, y en vista de (i) la degradación ambiental causada por la agricultura industrial y (ii) la advertencia de que nuestra alimentación, así como la calidad de los productos que consumimos, van deteriorándose paulatinamente conforme la denominada globalización económica avanzaba, muchos ciudadanos/consumidores han cambiado su mirada hacia los productos ecológicos, terminando por convertirse también en un gran modelo de negocio. Ya hemos comentado que el objetivo de las grandes empresas estriba en vender sus productos, que no en ser sinceros de cara al consumidor. $Y$ así comenzaron a surgir 0 redefinirse conceptos claves como "agricultura de conservación" o "agricultura orgánica". Sin embargo, estas últimos tampoco resultan ser sinónimos de agricultura ecológica, escondiéndose bajo vocablos aparentemente amigables prácticas e intereses que distan mucho de ser lo que el ciudadano profano considera "natural".

Si se analizan, por ejemplo, las actividades de la Asociación Española de Agricultura de Conservación, comprobarán el contenido estreñido de sus objetivos (en 2018), así como ninguna mención explícita de los principios ecológicos. Más aun, no es infrecuente que bajo diversas iniciativas y agrupaciones que apelan a tales vocablos, se oculten detrás relaciones muy estrechas con multinacionales que venden transgénicos, fertilizantes químicos y pesticidas (busquen en Internet la página Web de esta agrupación, o la de Monsanto). Eso sí, la FAO ha hecho un flaco favor al ciudadano al proponer un concepto de agricultura de conservación muy parecido al de agricultura ecológica. "la posverdad por 
delante" y que luego extraiga provecho el más "emprendedor".

Más difícil resulta comparar los objetivos de la agricultura orgánica ("organic farming") respecto a los de la ecológica, ya que el primer vocablo ha sido durante largo tiempo un aparente sinónimo del segundo en el mundo anglosajón, si bien sus principios son más relajados. Sustituir los fertilizantes minerales por la materia orgánica y evitar el uso de pesticidas y transgénicos, por desgracia, no son garantes de la producción de alimentos saludables. Por ejemplo, y como veremos más adelante, muchos lodos de depuradora, contienen elementos tóxicos, mal que nos pese. Su carga dependerá de donde procedan y como se composten s.l. las materias primas orgánicas que contienen. Empero lodos aparentemente "limpios" resultan no serlo, ya que los métodos utilizados nunca logran extraer todas las sustancias nocivas. Por ejemplo, a la hora de redactar este manuscrito, una noticia de prensa, alertaba que una buena parte de los lodos de depuradora utilizados se encontraban cargados de microplásticos, los cuales son nocivos para la salud y la biosfera. Los denominados contaminantes emergentes, indetectables por las depuradoras y el estudio de sus efectos para la salud, siguen siendo un quebradero de cabeza para nuestras autoridades sanitarias.

No debe tampoco soslayarse el procesamiento y venta de los productos. Así por ejemplo, mientras en ciertas marcas españolas se explicita el contenido en aceite de oliva en otras, generalmente germanas 0 anglosajonas, se juega y re-juega con las grasas mono-insaturadas, poli-insaturadas etc., con una frivolidad y ambigüedad que asustan.

Empero conforme la calidad de los alimentos decrece y la concienciación ambiental ciudadana aumenta, los vocablos han ido transmutándose según los intereses de mercado. Como corolario se ha abundado en la "ceremonia de la confusión", es decir la posverdad.

No es por tanto ridículo preguntarse: ¿en qué se diferencian las agriculturas orgánicas, de conservación y ecológica? Francamente todo depende de la fecha de la definición, el documento inspeccionado, y/o la página Web visitada. Topamos de nuevo con la postverdad.

\section{NECESIDAD Y EXIGENCIA DE UNA AGRICULTURA ECOLÓGICA A ESCALA PLANETARIA.}

Tras la celebración del Año Internacional de los Suelos, en 2015, avalado por la ONU y la $\mathrm{FAO}$, existe a nivel internacional una mayor concienciación por la alarmante pérdida y degradación de los suelos en todo el mundo. Sin embargo, la cobertura mediática y su eficacia ha calado de forma muy dispar según los países: por ejemplo en España, los medios de comunicación y nuestras autoridades soslayaron el tema, mientras que en EE.UU. la USDA y su máximo responsable publicaban notas de prensa semanalmente. El Lanzamiento de la "Alianza Mundial por el Suelo" de la FAO (GSP) y del "El Panel Técnico Intergubernamental de Suelos" de la ONU (ITPS) parece también haber estimulado la concienciación ciudadana, política y mediática. Una vez establecidas ambas iniciativas, su dialogo con otras similares concernientes a diferentes recursos naturales, hasta el momento, se encuentra dando algunos frutos, como veremos seguidamente. No obstante, los graves y muy dispares problemas de salud pública ocasionados por las prácticas de la agricultura industrial, posiblemente, hayan tenido aun un mayor impacto entre los lectores. Es bien sabido que todo lo concerniente a la salud despierta un gran interés entre los ciudadanos, con independencia de su procedencia. Finalmente, los suelos han ido calando entre los expertos del cambio climático, por cuanto 
finalmente la comunidad cientifica ha reconocido su vital importancia en el sistema climático, apareciendo diversos artículos relacionados con el tema en las revistas de mayor impacto (Science, Nature, PNAs, etc.). Sin embargo en lo concerniente a este último ítem es de lamentar que en la mayor parte de los artículos publicados, se echen en falta expertos e instituciones relevantes en las ciencias del suelo. Lamentablemente en la lectura de estos documentos abundan los errores, imprecisiones y el redescubrimiento de la dinamita que los edafólogos conocemos sobradamente.

En la primavera de 2018 (ver bibliografía recomendada) tras una reunión celebrada en Medellín (Colombia) el IPIBES (2018) publicó el documento titulado "Summary for policymakers of the thematic assessment of land degradation and restoration", así como varias notas de prensa, en las cuales se evaluaba el estado de los suelos del mundo. Algunos de los datos ofrecidos resultan ser estremecedores. Conforme a una de las mentadas notas de prensa que versaba sobre el documento del IPIBES (2018): "La degradación "crítica" del suelo, principal causa de la pérdida de especies, pone en riesgo a 3.200 millones de personas en el mundo y provocará grandes migraciones para 2050(...).

Así mismo, en la mentada reunión (Sexta Plenaria de la Plataforma Intergubernamental en Biodiversidad y Servicios Ecosistémicos -(IPBES-) se muestran informaciones relacionadas con el recurso suelo, algunas de las cuales son de vital importancia para el futuro de la agricultura ecológica.

(i) La degradación del suelo es posiblemente el aspecto ambiental que afecta en mayor medida a la población mundial, por cuanto dos de cada cinco personas se ven gravemente afectadas.

(ii) Los recursos edáficos proporcionan, de un modo u otro, el 95 por ciento de todos los alimentos indispensables con vistas a sostener a una población humana que aumenta rápidamente.

(iii) Una la agricultura insustentable, la minería, al actividad industrial, el sellado del suelo y el y el cambio climático empujan a la Tierra hacia un punto de no retorno que provocará conflictos y migraciones humanas masivas.

(iv) El informe, codirigido por Luca Montanarella, incide en los niveles críticos de degradación de los recursos edáficos, afectando conspicuamente a la biodiversidad, así como de los servicios ecosistémicos.

(v) El $75 \%$ de la edafosfera sufre signos de degradación en mayor o menor grado.

(vi) La pérdida de la fertilidad del suelo, la erosión y su alarmantemente creciente contaminación, salinización y acidificación, ha causado una pérdida continuada de productividad, aumentando la vulnerabilidad de una parte considerable de la población mundial. El suelo es un recurso natural que se encuentra casi agotado. Es decir apenas quedan suelos fértiles por explotar, mientras se degradan extensiones importantes de los que antaño también sí lo eran.

(vii) El deterioro de este recurso así como el cambio climático, provocarán una reducción de los cultivos estimada en un $10 \%$ globalmente, si bien puede alcanzarse cifras del $50 \%$ en las regiones más vulnerables, amenazando pues la seguridad alimentaria, la depuración de aguas, provisión/demanda energética y otros aspectos de la naturaleza esenciales para la humanidad.

Más aun, la ONU y la FAO, tras una reunión celebrada por la última en Roma, publicó una 
nota de prensa del 3 de abril de 2018, reconociendo que:

(i) La agricultura industrial imperante ha sido incapaz de paliar el hambre en el mundo, degradando buena parte de los recursos biosféricos.

(ii) La producción de alimentos en base a agroquímicos y maquinaria pesada, imperantes desde el final de la Segunda Guerra Mundial han aumentado la producción insuficientemente, y a costa de causar un inmenso daño al planeta.

(iii) En algunos países, los métodos de producción agropecuaria actual, están dejando a los campesinos sin un centavo (...) y en casos como el de India, la sociedad ha sido alarmada por "olas de suicidio campesino".

(iv) La adopción de la "agroecología", con sus efectos beneficiosos sobre la calidad del suelo, más económica para los agricultores y respetuosa con el medio ambiente, ayudaría a revertir la creciente inseguridad alimentaria. Es decir, la agricultura industrial debería dar paso a otra ecológica genuinamente sustentable.

(v) Aproximadamente, unos 30 países han adoptado un marco legislativo 0 regulatorio para facilitar el desarrollo de la agroecología.

Tales comentarios hubieran sido impensables hace tan solo un decenio, y debemos felicitarnos por ello. Sin embargo no debe olvidarse que, tanto en la ONU como en la FAO, los representantes de os Estados firman hermosas actas, convenciones, etc., que raramente son llevadas a la práctica y/o terminan por fructificar. Es decir, con el tiempo se convierten en posverdades. Del mismo modo, la agroindustria resulta ser uno de los lobbies más poderosos e influyentes del planeta, por lo que reaccionarán en consecuencia. $\quad Y$ como dice el refrán:
"Hechos son amores y no buenas razones". No obstante, un reconocimiento de tal guisa cambará el estado actual de las cosas, de tal modo que la agroecología dejará de ser percibida como un capricho de los ciudadanos acaudalados de ciertos países ricos, como han intentado reiteradamente acusar los defensores de la agroindustria a los consumidores de productos ecológicos, así como a los agricultores que se acogen a tal práctica.

Por otro lado, en nombre de la sustentabilidad, cambio climático y calidad del suelo se proponen (i) iniciativas aparentemente beneficiosas, pero que no lo son o (ii) simplemente resultan inviables. Pondremos tan solo un ejemplo de cada una.

En los últimos años, numerosas autoridades e instituciones promueven la agricultura periurbana, en la que los ciudadanos puedan cultivar sus propios productos, tan ecológicos como deseen. Pues bien, aun suponiendo que los interesados tengan la suficiente información con vistas a llevar a cabo tal actividad, nadie les informa que los suelos, especialmente los de las ciudades muy pobladas se encuentran, a menudo, contaminados siendo muy generalizada la polución procedente de las deposiciones atmosféricas. Dicho de otro modo, la calidad del aire resulta ser a menudo deplorable, sobrepasando los límites/umbrales de contaminantes orgánicos e inorgánicos que aconseja la Organización Mundial de la salud (OMS), y tales aerosoles son depositados en suelos y aguas. Obviamente, la agricultura ecológica periurbana es loable, empero requiere estudios previos y monitorizaciones de suelos y aire, con vistas a garantizar la salubridad de los alimentos producidos. Sin embargo raramente tal control es realizado por las autoridades. En urbes pequeñas y de escasa actividad industrial tal riesgo debería ser menor, que en las grandes y polucionadas. Empero no debe soslayarse que pueden darse vertidos incontrolados/ilegales, más 0 menos antiguos, susceptibles de causar serios 
problemas de salud pública. Cabe recordar, por ejemplo, que los suelos ricos en metales pesados parecen generar graves problemas oncológicos a los que hacen uso de los mismos (López-Abente et al. 2018; Núñez et al. 2018). Si ustedes teclean en su buscador de Internet simultáneamente los vocablos: urban, soil contamination, public health, encontrarán abundante información a este respecto.

La mayoría de los lectores sabrán que el COP21 lanzó durante en 2015 la denominada Iniciativa "4 por 1000". Su objetivo estriba en incrementar el contenido de carbono de los suelos en un 4 por mil anualmente, al objeto de ayudar a mitigar el cambio climático vía secuestro de carbono.
Pues bien ante la alegría de muchos, cabe señalar que se trata de una misión inviable. Ustedes pueden buscar en Internet la razón, o también en un post de mi bitácora (http://www.madrimasd.org/blogs/universo/20 16/08/02/147562/comment-page-1).

Personalmente no logro comprender si muchos de mis colegas reflexionan o tan solo buscan "fama y gloria". La viabilidad del "4 por 1000" no resulta ser más que propaganda institucional. Si algún día se pretende llevar a cabo se convertirá en una posverdad, a no ser que se interponga la hábil y versátil agroindustria con sus biochar, con el propósito de depredar pingues beneficios repartiendo a diestro y siniestro ese producto "milagroso", que no lo es tanto, I menos hasta la fecha.

\section{EL PASADO PUEDE SER LA CLAVE DEL FUTURO.}

"Mirando hacia el pasado para mejorar el futuro: Un viaje por las culturas precolombinas" es el título de un capítulo de libro que redacté para la SEAE, en el año 2015. A lo largo del Neolítico, las diferentes etnias y culturas que salpican todo el planeta se han topado con el reto de lograr unos sistemas agrosilvopastorales sustentables, ya que de no ser así desaparecían. Existe pues un bagaje cultural de nuestros antepasados que resultaría insensato soslayar. En aquel texto realizaba un breve e incompleto recorrido sobre las prácticas sustentables de aquél continente antes del denominado descubrimiento de América. Francamente, la variedad de tipos de manejo de suelos y cultivos adaptados a casi todos los ambientes era asombrosa. Se trata de un bagaje tan basto como fascinante y que puede y debe ser fuente de inspiración para el futuro. Urge pues generar plataformas, libros rojos, y otros medios en línea que ofrezcan a los interesados este patrimonio cultural de toda la humanidad. La única iniciativa del que este autor tiene noticia a este respecto es la de la FAO denominada: Herencia de los sistemas agri-culturales del mundo ("world's agri-cultural heritage systems" (http://www.fao.org/giahs/become- a-giahs/en/). Si bien se trata de una loable iniciativa que lleva en funcionamiento desde 2002, apenas ha tenido repercusiones y debería, tanto ser implementada con mayor esmero, como acompañada de otras más ambiciosas. No se trata exclusivamente de describir aquellos sistemas de manejo relevantes según los criterios de la FAO y los gobiernos. Aun reconociendo mi desconocimiento de cómo se eligen estos enclaves, en el mapa de su distribución (ver la página Web http://www.fao.org/giahs/become-a-giahs/es/) y pinchando en la pestaña "SIPAM en el mundo") resulta palmario un alarmante sesgo asiático, así como un soslayo prácticamente total, hasta la fecha, de continentes enteros. La infravaloración de los desarrollos logrados por la etnoagricultura en Suramérica y África, pone en evidencia los sesgos geopolíticos de estos programas. No cabe extrañar que otro sesgo geográficamente similar ocurra al visionar la distribución mundial de los Geoparques de la Unesco (lbáñez et al. 2019).

El ejemplo del biochar genera dudas acerca de cómo los científicos actualmente hacemos uso de los tópicos de moda, con 
vistas a publicar, más que de propiciar el progreso de la humanidad. Topamos una vez más con la Tecnociencia. Se trata de una historia fascinante desde que Francisco de Orellana, vislumbró indirectamente las denominadas culturas que generaron las denominadas "terras pretas do índio" en la primera exploración de los europeos por la Cuenca Amazónica. El tema del biochar indígena, fue tan publicitado por sus bondades y promesas, que dio lugar a un repentino crecimiento exponencial del número de publicaciones de al menos un decenio (ipublica o perece!). Sin embargo, tras tanto esfuerzo y financiación, aun no se han descubierto los secretos de aquella enmienda/corrector que transformaba suelos inferiles en otros altamente productivos. Eso sí, rápidamente comenzaron a patentarse productos que no logran emular las propiedades de la tecnología indígena bajo el "seudónimo" de biochar. De todo este asunto he redactado abundante material en mi bitácora. Lo realmente intrigante deviene en que nuestra sociedad tecnológica (y nosotros como expertos), tan ufana de su tecnología, sea incapaz de lograr encontrar aquella "receta milagrosa" Ilevada a cabo por culturas que distaban mucho de ser consideradas civilizaciones. Con toda sinceridad, este autor no sabe que pensar, tanto más cuando tal tipo de enmiendas orgánico-carbonosas, u otros similares, han sido reiteradamente descubiertas en otros continentes, siendo consideradas por los granjeros indígenas como elementos esenciales para mantener la sustentabilidad de sus sistemas agrarios, como es el caso del Nepal.

Sería un error pensar que uno o pocos tipos de manejo basados en la agroecología, solucionarán nuestros problemas. No suelen existir recetas milagrosas, sino ingenio y medios, según las circunstancias. Por ejemplo, con independencia de condicionamientos medioambientales, las prácticas de manejo agroecológico deben cambiar, según hablemos de productos para introducir en el mercado, tan solo para paliar el hambre y la pobreza de los más desheredados, o mantener a poblaciones aborígenes que, aunque acosadas por las sociedades modernas, desean permanecer al margen de ella, preservando así su identidad y modos de vida tradicionales.

\section{¿SABEMOS LO SUFICIENTE SOBRE LA ECOLOGÍA DEL SUELO?}

El suelo es un medio opaco difícil de analizar y más aún vislumbrar, conocer y apreciar en todo su asombroso entramado. Como en otras ramas de la ciencia del suelo, el estudio de la ecología de los sistemas edáficos ha progresado muy lentamente, por el escaso interés mostrado por nuestras autoridades a la hora de financiar tal tipo de investigaciones. Así, por ejemplo, no existe ningún suelo en el que se hayan estudiado todos los taxones biológicos que allí se encuentran. Personalmente considero que debemos aprender mucho más con vistas a extraer todas las enseñanzas que, a la postre, se aplicarían en las indagaciones agroecológicas futuras. No obstante, en los últimos años, existen señales estimulantes de que algo está cambiando. Pongamos tan solo dos ejemplos.

La Dra. Suzanne Simard, sigue una línea de investigación enormemente interesante mostrando en sus estudios y videos que la vida del suelo, los variados tipos de comunicación entre sus organismos, así como las inherentes relaciones con la parte aérea de los ecosistemas resulta ser casi tan asombrosa como la relatada en conocida película de Avatar (pueden consultar sus artículos en el repositorio de la autora en Researchgate). Una información más divulgativa puede encontrarse en el post de mi bitácora que lleva por título: "La Inteligencia de los bosques y su comunicación bajo el Suelo". En la misma 
dirección, apunta el estudio recientemente publicado por Zhalnina et al. (2018), por lo cual recomiendo a los lectores que lean sobre esta maravillosa perspectiva acerca de la ecología de los ecosistemas edáficos. Se trata de otro tema que necesita mucha mayor atención, por parte de los gestores de la actividad científica, incluyendo la implementación del instrumental necesario con vistas a conseguir penetrar en su interior y explorar sin perturbar. No obstante, cuando uno lee las noticias diarias que ofrece la ciencia al respecto, se me antoja desconcertante, que se dedique más dinero e interés en averiguar si existe/existió vida y agua en los suelos/regolitos marcianos, que en los terrestres, cuya estructura y dinámica tan solo comienza a vislumbrarse en su maravillosa plenitud. Debemos pues ser prudentes y reconocer tales conspicuas lagunas en nuestro conocimiento científico.

El método científico exige que los hallazgos de una indagación se corroboren por otros investigadores en una gran variedad de condiciones y ambientes, antes de que puedan alcanzar el estatus de teorías. La bibliografía actual y la comunidad científica no siguen lamentablemente las enseñanzas de esta disciplina, sino los dictados de la tecnociencia. Bajo el lema de publica 0 perece (publish or perish) la única verdad es que los expertos desean por notoriedad $y / 0$ simple supervivencia que les publiquen los artículos en revistas de "excelencia", y cuantos más mejor. No es usual que unos científicos intenten corroborar los resultados de otros, ya que no hay descanso entre la publicación de un artículo y el siguiente, el márquetin de los resultados en los medios de comunicación de masas y redes sociales, la búsqueda de financiación, etc. Tan solo si un descubrimiento resulta ser de gran importancia, al menos mediática, dará lugar a que otros colegas intenten corroborar/refutar los resultados. Como corolario, no seguimos los consejos del método científico. Y así, finalmente, es difícil discernir entre lo que resulta ser verdad, posverdad o simplemente falso ("fake news").

\section{A MODO DE CONCLUSIÓN: NECESITAMOS ALGO MÁS QUE CIENCIA, TECNOLOGÍA: VOLUNTAD POLÍTICA Y PUESTA EN ACCIÓN}

A modo de conclusión, debemos señalar que si deseamos extraer toda la potencialidad de que ofrece la agroecología en la producción de alimentos, sostenibilidad y preservación de la biosfera, queda mucho camino por recorrer. Personalmente, sería optimista si las recientes reacciones de organismos como la ONU y la FAO, tanto en materia de suelos como de agroecología calaran entre el público y los gestores naciones implicados en materias de política científica. Sin embargo, la historia de las iniciativas precedentes de esta guisa, no dan lugar para serlo. A pesar de todo, el mero hecho de que se reconozca el fracaso ambiental de la agricultura industrial, la insustentabilidad de nuestra contaminante sociedad y sus graves repercusiones sobre la salud pública, así como un genuino respaldo de las instituciones aludidas, tendrán como primer efecto el que edafólogos y agroecólogos no serán ninguneados en el futuro, mientras el lobby agroindustrial, tendrá más limitaciones para seguir vanagloriándose, de unas indagaciones que trajeron en su día más alimentos, pero a costa de poner tanto a la biosfera, como al hombre, al borde del precipicio. Y a la espera de lo que ocurra, científicos, instituciones y políticos, parchean nuestras lagunas de conocimientos y sentido común con posverdades. 


\section{REFERENCIAS}

Arana, M. 2009. La tecnociencia como "unidad del conocimiento". Colombia Revista Científica General José María Córdova 7 (7): 33-39.

Echeverria, J. 2003. La Revolución Tecnocientífica. Fondo de Cultura Económica, Madrid. ISBN: 9788437505510 (artículo resumen en acceso abierto).

Echeverria J. 2007. Ciencia del Bien y el Mal, Herder, Barcelona. ISBN: 9788425425073.

Echeverria J. 2010. De la filosofía de la ciencia a la filosofía de la tecnociencia Revista Internacional de Filosofía: 50, 2010, 31-41; ISSN: 1130-0507 (acceso abierto).

FAO, 2015, Status of the World's Soil Resources. Main Report. http://www.fao.org/3/a-i5199e.pdf.

Ibáñez, J. J., Ruiz Ramos, M., Zinck, J. A. and Brú, A. 2005. Classical pedology questioned and defended. Eurasian Soil Sci., 38. Suppl. 1, S75-S80.

Ibáñez, J.J. Krasilnikov, P., Saldaña, A., 2012. Archive and refugia of soil organisms: applying a pedodiversity framework for the conservation of biological and non-biological heritages. Journal of Applied Ecology, 49 (6): 1267$1277 . \quad$ https://doi.org/10.1111/j.13652664.2012.02213.x.

Ibáñez, J.J., (2015), Mirando hacia el pasado para mejorar el futuro: Un viaje por las culturas precolombinas. En: La Sociedad, la Agricultura y el Suelo. Tello, J.J. Jaizme-Vega, M.C. y Porcuna, J.L. (Eds). La Sociedad, La Agricultura y el Suelo. Sociedad Española de Agricultura Ecológica. SEAE gobierno de Canarias, pp.247-270. ISBN?. https://www.researchgate.net/publication/2863 79741_Mirando_hacia_el_pasado_para_mejor ar_el_futuroUn_viaje_por_las_culturas_precol ombinas.

Ibáñez, J.J., Brevik, E.C. \& Cerdá, A. 2019. Geodiversity and geoheritage: Detecting scientific and geographic biases and gaps through a bibliometric study. Science of The Total Environment 659: 1032-1044. https://doi.org/10.1016/j.scitotenv.2018.12.443.

Latour, B. 2001. La Esperanza de Pandora. Editorial Gedisa ISBN: 84-7432-787-3, Barcelona.

http://www.ub.edu/prometheus21/articulos/arc hivos/Tecnociencia.pdf.

López-Abente, G. Locutura-Rupérez, J., Fernández-Navarro, P., Martín-Méndez, I., Bel-Lan, A. Núñez, O., (2018), Compositional analysis of topsoil metals and its associations with cancer mortality using spatial misaligned data". Environmental. Geochemistry and Health 40: 283-294. DOI: 10.1007/s10653016-9904-3.

Lugschitz, B., Bruckner, M., Giljum, S. (2011), Europe's Global Land Demand: A study on the actual land embodied in European imports and exports of agricultural and forestry products. Final Report September 2011. Sustainable Europe Research Institute (SERI) Garnisongasse 7/17, A-1090 Vienna. https://www.foeeurope.org/sites/default/files/pu blications/europe_global_land_demand_oct11 1.pdf

Núñez, O, Fernández-Navarro, P., MartínMéndez, I., Bel-Lan, A., Locutura Rupérez, J., López-Abente. J. (2018), Association between heavy metal and metalloid levels in topsoil and cancer mortality in Spain". Environmental Science and Pollution Research International 24: 7413-7421, $2017 . \quad$ DOI: doi.org/10.1007/s11356-017-8418-6.

Würtenberger, L., Koellner, T. and Binder, C.R., ( 2006), Virtual land use and agricultural trade: Estimating environmental and socioeconomic impacts. Ecological Economics, 57: 679-697;

https://doi.org/10.1016/j.ecolecon.2005.06.004. 
Zhalnina, K., Louie, K, B., Hao, Z., Mansoori, N., Nunes da Rocha, U., Shengjing Shi, S., Cho, H., Karaoz, U., Loqué, D., Bowen, B. P., Firestone, M. K., Northen, T. R., Brodie; E. L. (2018), Dynamic root exudate chemistry and microbial substrate preferences drive patterns in rhizosphere microbial community assembly. Nature Microbiology, 3: 470-480. doi:10.1038/s41564-018-0129-3.

\section{REFERENCIAS RECOMENDADAS}

EC, 2018, Intergovernmental Science-Policy Platform on Biodiversity and Ecosystem Services. Distr.: Limited 24 March 2018 Original: English Plenary of the Intergovernmental Science-Policy Platform on Biodiversity and Ecosystem Services Sixth session Medellin, Colombia, 18-24 March 2018 Item 7 of the provisional agenda* Thematic assessment of land degradation and restoration. https://ec.europa.eu/jrc/en/scienceupdate/land-degradation-threatens-well-beingpeople-and-planet

FAO, 2015, Status of the World's Soil Resources. Technical Summary. ISBN: 97892-5-108960-6.

http://www.fao.org/documents/card/en/c/39bc9 f2b-7493-4ab6-b024-feeaf49d4d01/

FAO, 2017, Directrices voluntarias para la gestión sostenible de los suelos Organización de las Naciones Unidas para la Alimentación y la Agricultura Roma, Italia file://C:/Users/Juan\%20José\%20lbañez/Dropb
ox/GSP\%20Nota $\% 20$ Técnica/Directrices $\% 20 \mathrm{v}$ oluntarias $\% 20$ para $\% 20$ la\%20gestión\%20soste nible $\% 20$ de $\% 20$ los $\% 20$ suelos $\% 20$ FAO $\% 2020$ 16.pdf

IPBES (2018): Summary for policymakers of the thematic assessment report on land degradation and restoration of the Intergovernmental Science-Policy Platform on Biodiversity and Ecosystem Services. R. Scholes, L. Montanarella, A. Brainich, N. Barger, B. ten Brink, M. Cantele, B. Erasmus, J. Fisher, T. Gardner, T. G. Holland, F. Kohler, J. S. Kotiaho, G. Von Maltitz, G. Nangendo, R. Pandit, J. Parrotta, M. D. Potts, S. Prince, M. Sankaran and L. Willemen (eds.). IPBES secretariat, Bonn, Germany. https://reporterre.net/IMG/pdf/sols-

ipbes_re_sume_pour_les_de_cideursmars_2018.pdf.

IPBES, (2018), Worldwide Land Degradation and Restoration Assessment Report: A Primer. https://reliefweb.int/report/world/worldwideland-degradation-and-restoration-assessmentreport-primer-enarruzh.

Medina, M. (sin fecha): Tecnociencia (documento en acceso abierto). http://www.ub.edu/prometheus21/articulos/arc hivos/Tecnociencia.pdf

Medina, M. (sin fecha). La Cultura de la tecnociencia. (documento en acceso abierto). http://www.ub.edu/prometheus21/articulos/Cra TC\%AADF.pdf. 